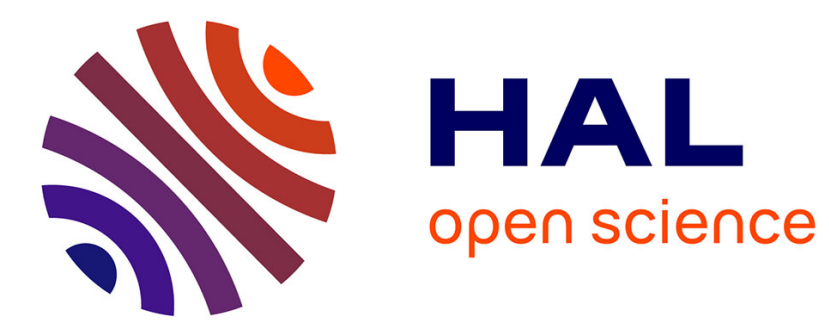

\title{
Dynamic resource allocation for Cloud-RAN in LTE with real-time $\mathrm{BBU} / \mathrm{RRH}$ assignment
}

Mohammed Yazid Lyazidi, Nadjib Aitsaadi, Rami Langar

\section{To cite this version:}

Mohammed Yazid Lyazidi, Nadjib Aitsaadi, Rami Langar. Dynamic resource allocation for CloudRAN in LTE with real-time BBU/RRH assignment. IEEE International Conference on Communications (ICC 2016), May 2016, Kuala Lumpur, Malaysia. 10.1109/ICC.2016.7511580 . hal-01357155

\section{HAL Id: hal-01357155 \\ https://hal.science/hal-01357155}

Submitted on 5 Sep 2016

HAL is a multi-disciplinary open access archive for the deposit and dissemination of scientific research documents, whether they are published or not. The documents may come from teaching and research institutions in France or abroad, or from public or private research centers.
L'archive ouverte pluridisciplinaire HAL, est destinée au dépôt et à la diffusion de documents scientifiques de niveau recherche, publiés ou non, émanant des établissements d'enseignement et de recherche français ou étrangers, des laboratoires publics ou privés. 


\title{
Dynamic Resource Allocation for Cloud-RAN in LTE with Real-Time BBU/RRH Assignment
}

\author{
Mohammed Yazid Lyazidi, Nadjib Aitsaadi* and Rami Langar \\ Sorbonne Universites, UPMC Univ Paris 06, CNRS, LIP6 UMR 7606, 4 place Jussieu 75005 Paris, France. \\ *LiSSi, University of Paris-Est Creteil Val de Marne (UPEC), 122 rue Paul Armangot, 94400 Vitry-sur-Seine, France \\ Emails: yazid.lyazidi@lip6.fr, nadjib.aitsaadi@u-pec.fr, rami.langar@lip6.fr
}

\begin{abstract}
Cloud-Radio Access Network (C-RAN) is a new emerging technology that holds alluring promises for Mobile network operators regarding capital and operation cost savings. However, many challenges still remain before full commercial deployment of C-RAN solutions. Dynamic resource allocation algorithms are needed to cope with significantly fluctuating traffic loads. Those algorithms must target not only a better quality of service delivery for users, but also less power consumption and better interference management, with the possibility to turn off RRHs that are not transmitting. To this end, we propose in this paper a dynamic two-stage design for downlink OFDMA resource allocation and BBU-RRH assignment in C-RAN. Specifically, we first model the resource and power allocation problem in a mixed integer linear problem for real-time fluctuating traffic of mobile users. Then, we propose a Knapsack formulation to model the BBU-RRH assignment problem. Simulation results show that our proposal achieves not only a high satisfaction rate for mobile users, but also minimal power consumption and significant BBUs savings, compared to state-of-the-art schemes.
\end{abstract}

Keywords: Cloud-RAN, LTE, Resource allocation, Power minimization, BBU reduction.

\section{INTRODUCTION}

Cloud-Radio Access Network, commonly known as C-RAN, has been introduced by [1] as a new cloud architecture for future Mobile Network Operators (MNOs) infrastructure. C-RAN's architecture is illustrated in Fig. 1. It is based on a centralized baseband pool regrouping many BaseBand Units (BBUs), which are composed of high-performance programmable processors and real-time virtualization technology that perform baseband (PHY/MAC) processing. BBUs are deployed on general purpose platforms and are connected via a high bandwidth low-latency optical network to distributed Radio Remote Heads (RRHs), which are located at the remote site. A Cloud controller [2], located in the BBU pool, serves as a resource manager and performs load balancing between cloud base stations to accommodate the traffic load of different cells. This centralized architecture provides operators better flexibility in network dimensioning, performed adaptability to non-uniform traffic and efficient utilization of baseband resources.

However, there are several challenges that need to be addressed before full commercial deployment of C-RAN solutions. First of all, a high-bandwidth low latency optical network is needed to transmit the RRH baseband data in an optical link from the BBU pool to the cell site. Due to the strict LTE timing requirements of $1 \mathrm{~ms}$ for physical layer processing [3], a transmission link with at least $10 \mathrm{Gbit} / \mathrm{s}$ with tight constraints on transmission time and latency is required [4]. On another side, RRHs must be properly assigned to BBUs, not

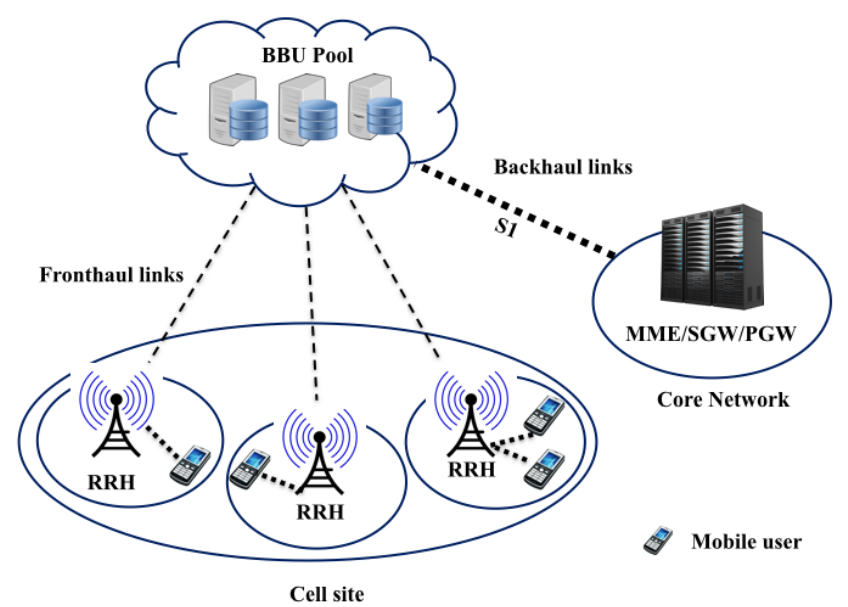

Fig. 1: Cloud Radio Access Network (C-RAN) LTE Architecture

only to facilitate collaboration techniques such as Cooperative Multipoint Processing (CoMP), but also to prevent the BBU Pool and the transport network from getting overloaded. A suitable assignment must be done between BBUs and RRHs regarding interference management, BBU pool capacity and traffic demand. Moreover, to optimize energy savings of CRAN, some RRHs need to be chosen to be turned on/off in a way that will lessen the overhead in the transport network and reduce the number of active BBU units within the BBU Pool.

In this context, we propose in this paper a two-stage design for Dynamic Resource Allocation in C-RAN, namely DRAC, with real-time BBU-RRH assignment, considering the constraints in transmission power and Signal-to-Interferenceplus-Noise-Ratio (SINR) for User Equipments (UEs). To the best of our knowledge, we believe that this paper is the first attempt to tackle both the resource allocation and BBURRH assignment problems in one framework. Specifically, we investigate how to dynamically optimize the set of active RRHs and allocated resources to serve real-time traffic of incoming UEs. To do so, we first propose a resource allocation algorithm that associates dynamically the best spectrum set of frequency/time resources to incoming UEs, with proper UE-RRH attachment. The problem is first formulated as nonconvex and NP-hard, then linearized into Mixed Linear Integer Problem (MILP), and solved using a Branch \& Cut (B\&C) algorithm. Based on the results of the first phase, the second stage consists of computing the optimal number of BBUs 
required to manage the system, and appropriately assigning them to RRHs in order to handle the whole traffic load. We model this second problem as a Multiple Knapsack Problem (MKP), which can be efficiently solved by modern linear solvers such as IBM CPLEX [5].

To gauge the effectiveness of our proposal, we compare our resource allocation design with two existing strategies in centralized resource allocation and power control: the QPFCRA approach [6] and GSB Algorithm [7]. For the BBURRH assignment problem, we compare our solution to the Semi-static and Adaptive schemes proposed in [8].

The remainder of this paper is organized as follows. In Section II, we review some recent and noteworthy related works on C-RAN. Section III describes the mathematical characterization of our two-stage approach. In Section IV, we present the $\mathrm{B} \& \mathrm{C}$ algorithm used to implement our approach and solve the resource allocation problem. Numerical results are presented in Section V to illustrate the performance of DRAC. Finally, Section VI concludes this paper.

\section{RELATED WORK}

There have been plenty of active research efforts on C-RAN after its recent introduction. Authors in [4] presented a detailed survey regarding the state-of-the-art literature of C-RAN while highlighting its advantages for operators compared to traditional Base Stations (BS). What's striking, while more BSs are needed to increase mobile traffic coverage, some of them are underutilized during certain hours in the day that correspond to low traffic load, resulting in ineffective use of baseband resources. C-RAN can address this issue, as motivated in [4] and [9] by dynamically setting the BBU-RRH connections and allocating resources to RRHs according to users' traffic profiles and volumes. As a result, cost reduction and energy savings can be achieved due to the reduction in the number of BBUs and effective utilization of the baseband resources.

Authors in [6] presented the QP-FCRA framework, which is a centralized approach for resource and power allocation in femtocells networks that can be applied in C-RAN context. In their proposal, cooperation between neighboring RRHs is exploited to improve resource allocation and throughput satisfaction via power minimization. Within each cluster of RRHs, a joint resource and power allocation is centralized at a cluster-head that periodically optimizes the throughput satisfaction. However, real-time resource allocation considering users arrival and departure was not taken into account.

In [7], the authors presented two Group Sparse Beamforming (GSB) algorithms, to minimize the whole C-RAN power consumption. Their proposal includes the transport network power and the power transmitted from the RRHs, with a SINR constraint at each user. The authors presented the problem as a joint RRH selection and transmit power minimization beamforming problem. They have addressed this problematic by sorting RRHs in an increasing order of their beamforming gain (i.e., transmitting power). The algorithm successively switches off RRHs with minimum beamforming gain until the problem becomes infeasible to minimize the whole power consumption. However, their approach does not exhibit the number of BBUs required to manage the entire system. In fact, their study has been carried out separately, without taking into account the reconfigurations that can be performed at the BBU pool level.

Based on the traffic analysis of Tokyo's metropolitan area, the authors in [10] proposed a new Colony RAN architecture that can reduce the number of BBUs by maximum $75 \%$, compared to the traditional RAN architecture. This nevertheless remains a rough estimation by the authors themselves. In [8], the same authors proposed two BBU-RRH switching schemes for C-RAN: semi-static and adaptive. The semi-static algorithm determines the combinations of BBUs and RRHs to accommodate peak hour traffic load for all RRHs within a large time interval. In contrast, their adaptive scheme assigns RRHs to BBUs based on neighboring RRHs loads and BBUs resource usage limits within a short time interval (one hour). They demonstrated that under a traffic distribution in an office area, the number of BBUs can be reduced by $26 \%$ and $47 \%$ for the semi-static and adaptive schemes, respectively. However, their approaches did not take into account that RRHs can be turned off depending on traffic load fluctuation during a time interval.

\section{Mathematical Models}

We consider a densely deployed C-RAN in a large area consisting of $S$ RRHs denoted by the set $\mathcal{S}=\{i \mid 1 \leqslant i \leqslant S\}$. We denote by $\mathcal{K}=\{k \mid 1 \leq k \leq K\}$ the set of available Physical Resource Blocks (PRBs) jointly assigned by the BBU pool to all $S$ RRHs.

\section{A. Downlink Resource Allocation Problem Formulation}

As a first step, we focus on the resource allocation problem for a real-time fluctuating traffic of static mobile users. We model UEs' arrivals with a Poissonian process where each arriving UE $u$ will be attached and served by one RRH $i$. User demands represent the required bandwidth, and can be expressed in number of required PRBs $N_{u}$. Our goal is to find the optimal resource allocation strategy - i.e., to find the best-serving RRHs and best PRBs allocation in downlink, while minimizing the gap between the required and allocated resources. Note that, the transmission power from RRH $i$ to UE $u$ can be independently allocated on each assigned PRB $k$. In fact, dynamic or fixed power allocation can be performed depending on the channel's variation speed [11]. We define our PRB allocation, user's attachment to RRH and transmit power allocation using the following variables:

$$
\begin{aligned}
y_{i k}^{u} & = \begin{cases}1, & \text { if PRB } k \text { is allocated to } \mathrm{UE} u \text { on RRH } i \\
0, & \text { otherwise. }\end{cases} \\
x_{i}^{u} & = \begin{cases}1, & \text { if } \mathrm{UE} u \text { is attached to } \mathrm{RRH} i, \\
0, & \text { otherwise. }\end{cases} \\
p_{i k}^{u} & = \begin{cases}p \in\left(0, P_{\text {max }}\right], & \text { if } y_{i k}^{u}=1, \\
0, & \text { otherwise. }\end{cases}
\end{aligned}
$$

The SINR achieved by UE $u$, attached to RRH $i$ and on a given PRB $k$ can be written as:

$$
\Gamma_{i k}^{u}=\frac{p_{i k}^{u} g_{i k}^{u}}{\sum_{j \neq i} \sum_{v \neq u} p_{j k}^{v} g_{j k}^{u}+\sigma^{2}}
$$

Where $g_{i k}^{u}$ is the path gain between RRH $i$ and UE $u$, and $\sigma^{2}$ is the noise power. Let $\mathcal{N}$ denote the set of time events 
corresponding to a UE's arrival and $\mathcal{U}_{n}$ the set of existing UEs in the system at event $n \in \mathcal{N}$. Our optimization problem can be written as follows:

$$
\begin{aligned}
\min _{x^{u}, y^{u}, p^{u}} & f_{n}^{(1)}=\sum_{u \in \mathcal{U}_{n}} \sum_{i \in \mathcal{S}} \sum_{k \in \mathcal{K}} \alpha \frac{p_{i k}^{u}}{P_{\max }}-(1-\alpha) \frac{x_{i}^{u} y_{i k}^{u}}{K} \\
\text { subject to } & \sum_{i \in \mathcal{S}} \sum_{k \in \mathcal{K}} x_{i}^{u} y_{i k}^{u} \leqslant N_{u}, \forall u \in \mathcal{U}_{n} \\
& \sum_{i \in \mathcal{S}} x_{i}^{u} \leqslant 1, u \in \mathcal{U}_{n} \\
& \sum_{u \in \mathcal{U}_{n}} \sum_{k \in \mathcal{K}} p_{i k}^{u} \leqslant P_{\text {max }}, i \in \mathcal{S} \\
& \Gamma_{i k}^{u} \geqslant y_{i k}^{u} \gamma_{u}, i \in \mathcal{S}, k \in \mathcal{K}, u \in \mathcal{U}_{n} \\
& p_{i k}^{u} \geqslant y_{i k}^{u} p_{\text {min }}^{u}, i \in \mathcal{S}, k \in \mathcal{K}, u \in \mathcal{U}_{n} \\
& \forall(u, v) \in \mathcal{U}_{n}^{2}, y_{i k}^{u}+y_{i k}^{v} \leqslant 1, i \in \mathcal{S}, k \in \mathcal{K} \\
& y_{i k}^{u} \leqslant x_{i}^{u}, i \in \mathcal{S}, k \in \mathcal{K}, u \in \mathcal{U}_{n} \\
& x_{i}^{u}, y_{i k}^{u} \in\{0,1\}, i \in \mathcal{S}, k \in \mathcal{K}, u \in \mathcal{U}_{n}
\end{aligned}
$$

Where $\alpha$ is a constant optimization weight between 0 and 1. Constraint (6) stresses the fact that the total allocated resources for each UE cannot exceed its requested demand $N_{u}$. Constraint (7) denotes that a UE can only be served by at most one RRH. (8) and (10) are the power constraints on RRH and UE, respectively, where $P_{\max }$ is the maximum transmission power for each RRH and $p_{\min }^{u}$ is the minimum power that can be transmitted to UE $u$. (9) is the SINR constraint where $\gamma_{u}$ refers to UE's $u$ individual SINR threshold. (11) ensures that two UEs attached to the same RRH cannot use the same PRB, (12) imposes all $y_{i k}^{u}=0$ if $x_{i}^{u}=0$, i.e., the RRH $i$ is not transmitting any PRBs, and finally (13) refers that $y_{i k}^{u}$ and $x_{i}^{u}$ are binary variables.

It is worth noting that the optimization problem in (6) is a Mixed Integer Non-Linear Program (MINLP), which is NPhard due to the quadratic objective function $f_{n}$ and the nonconvex SINR constraint (9) [12]. To simplify the resolution of this problem, we reformulate it as a MILP thanks to the big- $M$ method [13]. In fact, we can replace the product of the two binary variables $y_{i k}^{u}$ and $x_{i}^{u}$ by a new binary variable $z_{i k}^{u}$ and add the new following constraints:

$$
\begin{aligned}
& z_{i k}^{u} \leqslant x_{i}^{u}, \\
& z_{i k}^{u} \leqslant y_{i k}^{u}, \\
& z_{i k}^{u} \geqslant x_{i}^{u}+y_{i k}^{u}-1 .
\end{aligned}
$$

For the SINR constraint, we can rewrite it as follows:

$$
\left(1+\frac{1}{\gamma_{u}}\right) p_{i k}^{u} g_{i k}^{u} \geqslant y_{i k}^{u} \Omega_{k}^{u}+y_{i k}^{u} \sigma^{2}
$$

Where $\Omega_{k}^{u}$ is equal to $\sum_{j} \sum_{v} p_{j k}^{v} g_{j k}^{u}$. The non-convex product between binary variable $y_{i k}^{u}$ and continuous variable $\Omega_{k}^{u}$ can also be linearized using the big- $M$ modeling, providing we know $\Omega_{k}^{u}$ s lower and upper bounds. From (3) and (10) we can easily deduce $L$ and $U$, the lower and upper bounds of $\Omega_{k}^{u}$, respectively. Thus, the product $y_{i k}^{u} \Omega_{k}^{u}$ can be replaced by a new continuous variable $w_{i k}^{u}$ and the corresponding constraints can be rewritten as:

$$
\begin{aligned}
y_{i k}^{u} L & \leqslant w_{i k}^{u} \leqslant y_{i k}^{u} U \\
\left(1-y_{i k}^{u}\right) L & \leqslant \Omega_{k}^{u}-w_{i k}^{u}
\end{aligned}
$$

Hence, the MILP formulation of our DRAC problem can be expressed as follows:

$$
\begin{array}{ll}
\min _{x^{u}, y^{u}, p^{u}} & f_{n}^{(2)}=\sum_{u \in \mathcal{U}_{n}} \sum_{i \in \mathcal{S}} \sum_{k \in \mathcal{K}} \alpha \frac{p_{i k}^{u}}{P_{\max }}-(1-\alpha) \frac{z_{i k}^{u}}{K} \\
\text { subject to } & \sum_{i \in \mathcal{S}} \sum_{k \in \mathcal{K}} z_{i k}^{u} \leqslant N_{u}, \forall u \in \mathcal{U}_{n} \\
& (7),(8),(10),(11),(12),(13) \\
& (14),(15),(16) \\
& \left(1+\frac{1}{\gamma_{u}}\right) p_{i k}^{u} g_{i k}^{u} \geqslant w_{i k}^{u}+y_{i k}^{u} \sigma^{2} \\
& (18),(19)
\end{array}
$$

\section{B. RRH-BBU Assignment}

In conventional RRH-based RAN system, one BBU is used per RRH to handle the total traffic load. Thanks to C-RAN's centralization, the resource of one BBU can be shared between different connected RRHs that have few traffic load [10]. For instance, if a remote site is covered by 5 RRHs and each $\mathrm{RRH}$ has $20 \%$ of traffic load, one BBU is enough to manage all five RRHs. We can compute the optimal number of BBUs $B_{n}$ needed at each epoch $n$ to manage the whole system as follows:

$$
B_{n}=\left\lceil\frac{\text { Total Charge of the C-RAN }}{K}\right\rceil
$$

Where $\lceil$.$\rceil is the ceiling function and K$ is the total number of available PRBs in the system.

Our goal in this second stage is to properly assign RRHs to BBUs using a Multiple Knapsack Problem (MKP) formulation [14] where the objects are the RRHs and the Knapsack is the BBU capacity to handle the real-time traffic load. This assignment can be dynamically performed following the traffic load evolution at each epoch $n$. From the results of the first phase, we can collect the load of each RRH $i$ that we note $\left.\left.c_{i} \in\right] 0,1\right]$, corresponding to the weight of active RRH $i$, and the vector $\mathbf{c}=\left[c_{1}, \ldots, c_{i}, \ldots, c_{S}\right]$ corresponding to all $S$ RRHs' traffic load. The Knapsack's capacity is then defined as the optimal number of BBUs calculated in (25). We introduce a new binary variable $r_{i j}$, which is equal to one if RRH $i$ is attached to BBU $j$ and zero otherwise. Our BBU-RRH MKP problem will be thus formulated as follows:

$$
\begin{array}{ll}
\underset{r}{\operatorname{maximize}} & \sum_{j=1}^{B_{n}} \sum_{i=1}^{S} r_{i j} \\
\text { subject to } & \sum_{i=1}^{S} c_{i} r_{i j} \leqslant 1, j \in\left\{1, \ldots, B_{n}\right\} \\
& \sum_{j=1}^{B_{n}} r_{i j} \leqslant 1, i \in\{1, \ldots, S\}, \\
& r_{i j} \in\{0,1\}, i \in\{1, \ldots, S\}, j \in\left\{1, \ldots, B_{n}\right\}
\end{array}
$$


Constraint (28) denotes that one RRH cannot be attached to more than one BBU.

\section{Proposal: DRAC Algorithm}

In this section, we present our Dynamic Resource Allocation in C-RAN (DRAC) approach to solve the afore-mentioned problem formalized in (20). It is based on a B\&C algorithm, which is executed at each UE's arrival.

The Branch and Cut Algorithm [15] combines the advantages of Branch and Bound and Gomory Cutting Planes Schemes into one algorithm, that is not only reliable, but also much faster than Branch and Bound alone. The algorithm is based on a linear relaxation of integer variables into continuous ones, while adding Cutting Planes to improve the problem relaxation and come closer to approximate integer solutions. Considering our MILP problem formalized in (20), its variables are $z_{i k}^{u}, x_{i}^{u}, y_{i k}^{u}, p_{i k}^{u}$, and $w_{i k}^{u}$, which all contribute in a very huge optimization problem. Solving (20) with the B\&C scheme will seemingly take a fair amount of time, that will undoubtedly exceed the strict LTE $1 \mathrm{~ms}$ of timing constraint. Nevertheless, having a closer look at these variables, we remark that they are interdependent, in a way that variable $z_{i k}^{u}$ constitutes a "core" variable while the other ones can be derived from this core variable. In fact, variables $x_{i}^{u}$ and $y_{i k}^{u}$ can be derived from the big- $M$ reformulation constraints (14), (15), (16), and $p_{i k}^{u}$ comes as a "sub-core" optimization variable which can be deduced from (7) and (10). Consequently, we can focus our resolution on a smaller optimization space, generated by the core variable $z_{i k}^{u}$. Also, due to delay constraints imposed by real-time processing, we define in our B\&C algorithm an upper bound itermax on the number of iterations to reduce the computing time and to get closer to the processing time within the BBU pool's Cloud controller. The pseudo-code of the proposed PRB resource allocation problem is summarized in Algorithm 1, which is executed at each new UE's arrival.

Once the resources are allocated to incoming users, the next step is to optimize the BBU-RRH assignment by solving the MKP in (26). We used IBM's linear solver CPLEX, which was able to find optimal results with very low computation time (around $3 \mathrm{~ms}$ at each epoch).

\section{Performance Evaluation}

In this section, we evaluate the performance of our proposed DRAC framework and compare the benefits of our solution with respect to state-of-the-art schemes: QP-FCRA [6] and Iterative GSB Algorithm [7] for the resource allocation problem, as well as Semi-static and Adaptive switching algorithms [8] for the BBU-RRH assignment problem. Simulations are conducted on MATLAB, where we simulated a wireless LTE environment consisting of 16 RRHs, each one at the center of a hexagonal cell. The distance between two nearest RRHs is $100 \mathrm{~m}$. We considered the following channel model: $h_{i}^{u}=10^{-L\left(d_{i}^{u}\right) / 20} \sqrt{\phi_{i}^{u} s_{i}^{u}} g_{i}^{u}$, where $L\left(d_{i}^{u}\right)$ is the path-loss at distance $d_{i}^{u}$ between RRH $i$ and UE $u, \phi_{i}^{u}$ is the antenna gain, $s_{i}^{u}$ is the shadowing coefficient, and $g_{i}^{u}$ is the fading coefficient. We used IBM's linear solver CPLEX as well as the modeling tool YALMIP [16] to develop our algorithms. We generated 60 simulation runs for two scenarios of SINR threshold $\gamma$ : 10 and $25 \mathrm{~dB}$.

Algorithm 1 Downlink PRB Resource Allocation Algorithm

Inputs: Number of existing UEs $u$ with their demand $N_{u}$ and position at each arrival epoch $n$

Outputs: matrix $z$

I. Initialization:

1. Denote the initial problem $P^{0}$ and the set of active problem nodes to be $L=\left\{P^{0}\right\}$.

2. Let the initial value set of core variable $z^{*}=\emptyset$ and the initial lower bound $L B=-\infty$. Set $f^{*}=-\infty$ the initial value of objective function.

\section{Iteration: do}

while Number of iterations $\leq$ itermax do

3. Select and delete a problem $P^{l}$ from $L$.

4. Solve $P^{l R}$, relaxed version of $P^{l}$, where $\mathrm{z}$ takes continuous values between 0 and 1 .

5. if $P^{l R}$ is infeasible, go back to step 3. else denote the optimal solution $z^{l R}$ with objective function value $f$.

6. if $f \leq f^{*}$ go back to step 3 .

7. if $z^{l R}$ is integer, set $f^{*} \leftarrow f$ and $z^{*} \leftarrow z$. Go back to step 3 .

8. If desired, search for cutting planes from previous dropped constraints that are violated by $z^{l R}$; if any are found, add them to the relaxation and return to step 3 .

9. Branch to partition the problem into new problems with restricted feasible regions. Add these problem to $L$ and go back to step 3 .

10. Go to the next iteration.

\section{end while}

\begin{tabular}{c|c}
\hline Parameters & Values \\
\hline Number of RRHs & 16 \\
Bandwidth & $10 \mathrm{MHz}$ \\
Total number of PRBs & 50 \\
Max power $P_{\text {max }}$ & $20 \mathrm{~mW}$ \\
Min power $P_{\text {min }}$ & $0.1 \mathrm{~mW}$ \\
Constant $\alpha$ & 0.5 \\
Path loss model & $148.1+37.6 \log _{10}(d), d$ in Km \\
Shadowing standard deviation & $5 \mathrm{~dB}$ \\
Fading model & Normal distribution $\mathcal{N}(0, \mathbf{I})$ \\
Thermal noise & $-174 \mathrm{dBm} / \mathrm{Hz}$ \\
Transmit antenna power gain & $8 \mathrm{dBi}$ \\
Poisson Parameter & $\lambda \in[1,10](\mathrm{default} 1)$ \\
Departure rate & $\mu=0.1$ \\
UE's PRB demand & Uniform distribution $\mathcal{U}(1,10)$ \\
\hline
\end{tabular}

Table I: Simulation Parameters

For a fixed arrival rate of mobile users $(\lambda \in[1,10])$, we vary at each run their stay time and service demand following an Exponential and Uniform law, respectively. Note that UEs' positions are randomly generated at each run and remain fixed during their whole stay time in the network. The service demand of each user is expressed in terms of number of PRBs from a downlink LTE frame of 50 PRBs. We fixed the itermax metric of the $\mathrm{B} \& \mathrm{C}$ algorithm to 200 iterations. Table I reports the simulation parameters. In what follows, we present the corresponding simulation results in terms of Throughput Satisfaction Rate (TSR), Spectrum Spatial Reuse (SSR), transmitted power per RRH, number of BBUs, along with the active number of RRHs.

1) Throughput Satisfaction Rate (TSR): Fig. 2 shows the Cumulative Distributed Function (CDF) of the TSR, which is 


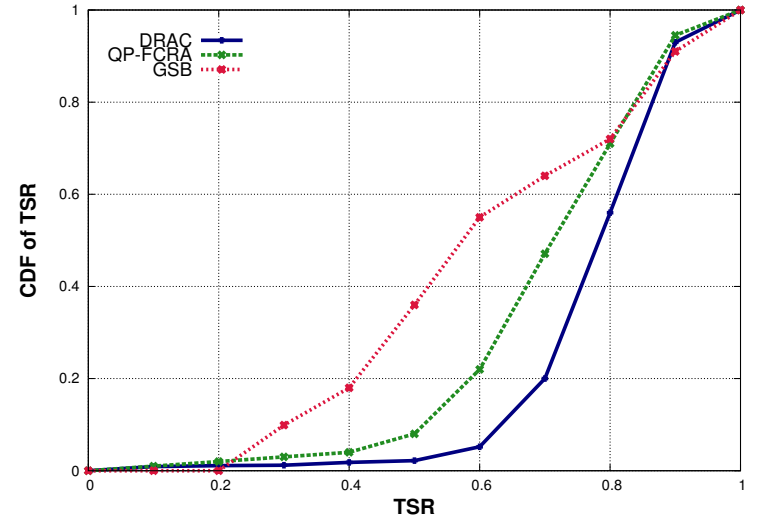

a) $\mathrm{SINR}=10 \mathrm{~dB}$

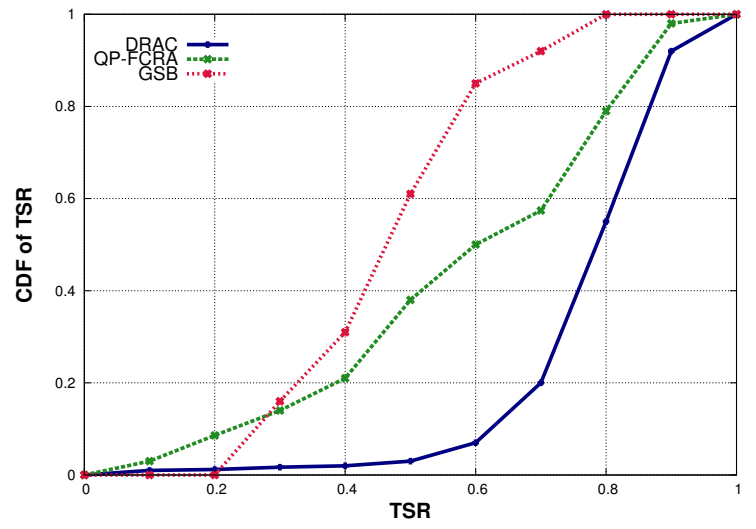

b) $\mathrm{SINR}=25 \mathrm{~dB}$

Fig. 2: Throughput Cumulative Density Function

Table II: Mean Spectrum Spatial Reuse

\begin{tabular}{|c|c|c|c|}
\hline SINR & DRAC & QP-FCRA & GSB \\
\hline $10 \mathrm{~dB}$ & 1.2256 & 0.4602 & 1.0118 \\
\hline $25 \mathrm{~dB}$ & 1.2152 & 0.4577 & 1.0076 \\
\hline
\end{tabular}

the ratio of the number of allocated PRBs to the total initial demands $N_{u}$. As we can observe, more than $50 \%$ of UEs have their TSR greater than $75 \%$ in both SINR thresholds for DRAC. The TSR is lessened to $63 \%$ and $55 \%$ for QPFCRA and GSB, respectively - as shown in Fig.2-a - at low SINR threshold, and to $60 \%$ and $44 \%$, respectively, in Fig. $2-$ b, when the SINR threshold is high. Hence, our proposed DRAC approach outperforms both QP-FCRA and GSB schemes.

2) Spectrum Spatial Reuse (SSR): Table II reports the SSR of the three aforementioned approaches. The SSR represents the average portion of RRHs transmitting the same PRB within the C-RAN system. The more a PRB is reused, the better is the performance. This is clearly shown in Table II, where DRAC fosters more PRBs reuse, by a factor of 2.6632 and 1.2113 compared to QP-FCRA and GSB approaches, respectively, at low SINR threshold. When the SINR threshold is high, the reuse factor is enhanced by 2.6550 and 1.2060 , respectively.

3) Transmitted Power per RRH: Fig. 3 illustrates the average power transmitted from each RRH. The GSB scheme performs the minimum power consumption by switching off RRHs based on their successive RRH selection algorithm. However, this is negatively reflected on the TSR of mobile

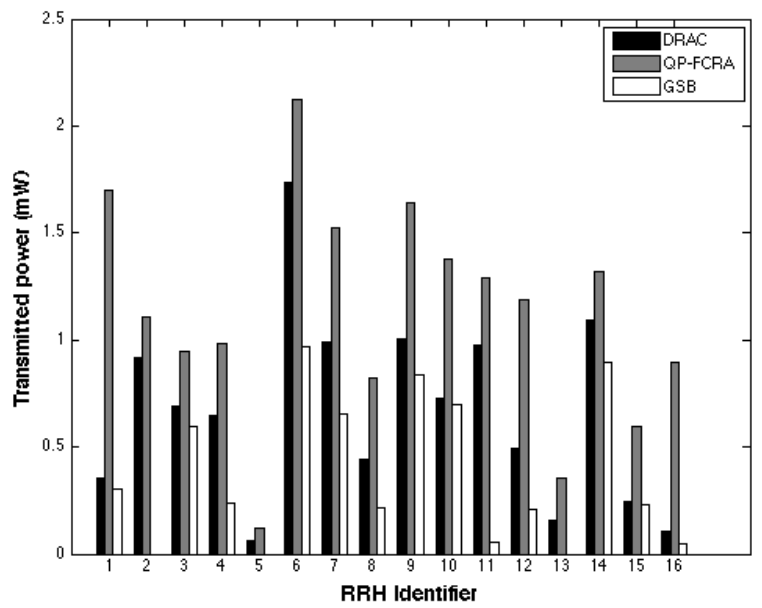

a) $\mathrm{SINR}=10 \mathrm{~dB}$

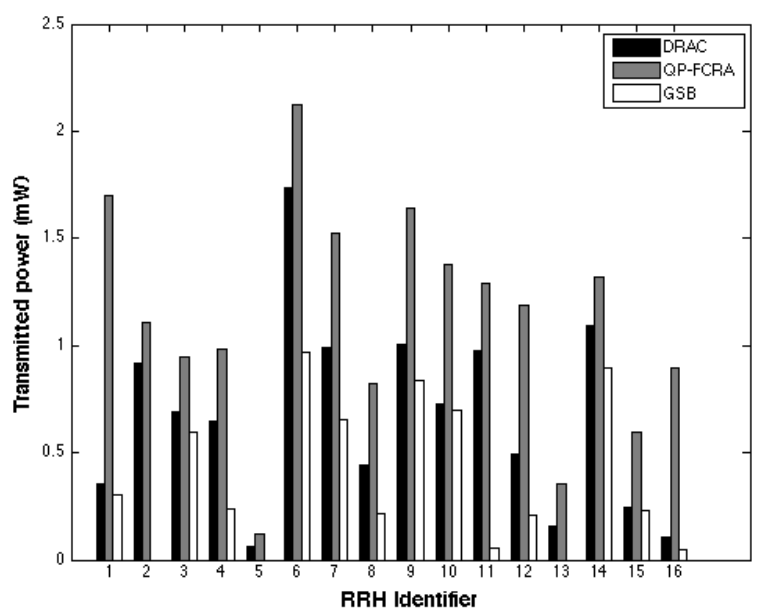

b) $\mathrm{SINR}=25 \mathrm{~dB}$

Fig. 3: Transmitted power per RRH

users, as seen in Fig. 2, since they are less satisfied by their allocated PRBs. In fact, while the iterative GSB method fosters more RRHs switching off, it will result in less transmission power consumption in the system, but will severely impact the throughput satisfaction for mobile users due to the dynamic scaling between power $p_{i k}^{u}$ and PRB allocation $y_{i k}^{u}$ variables. The QP-FCRA approach, on the other hand, supposes all RRH are turned on, which leads to a higher power consumption but to a good TSR. As observed in Fig.3 -a and -b, our proposed DRAC scheme performs a good tradeoff between satisfaction rate and overall power consumption for both SINR threshold levels, since our method finds the optimal balance of RRHs to be switched on/off to satisfy mobile users to their best, while minimizing the power consumption in the system.

4) Number of BBUs and On RRHs: Fig. 4 illustrates the number of BBUs needed per time for the conventional RAN, the RRH-based RAN and the C-RAN contexts, when the SINR threshold is equal to $25 \mathrm{~dB}$. The one-one mapping in conventional RAN imposes as many BBUs as deployed RRHs to handle the radio site coverage. RRH-based RAN represents the number of on RRHs based on our proposal to serve the 


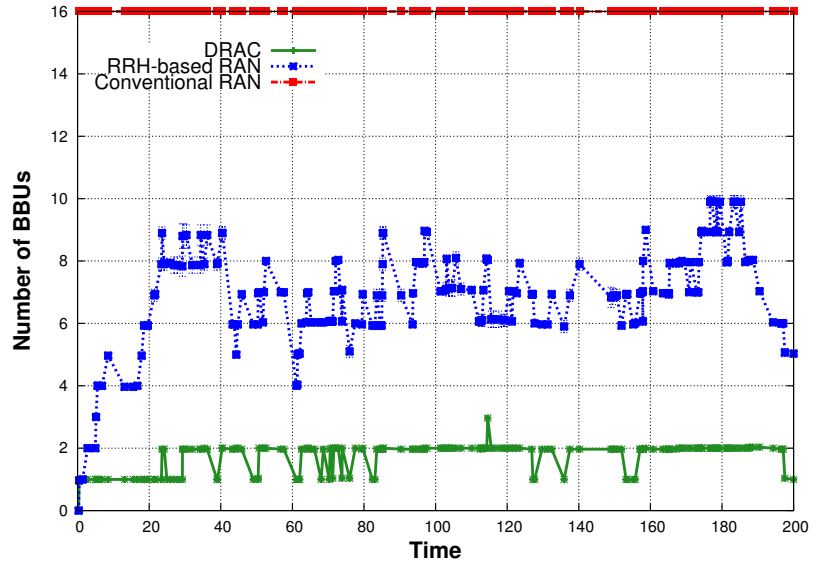

Fig. 4: Number of needed BBUs per time

Table III: Number of BBUs and RRHs

\begin{tabular}{|c|c|c|c|c|c|c|}
\hline Scheme & Mean BBU & $\min$ & $\max$ & Mean RRHs & $\min$ & $\max$ \\
\hline DRAC & 1.63 & 1 & 3 & 6.54 & 1 & 5.6 \\
\hline Semi-static & 11.74 & 10 & 14 & 16 & 1.6 & 2.1 \\
\hline Adaptive & 8.8 & 8 & 10 & 16 & 1.44 & 4.75 \\
\hline
\end{tabular}

fluctuating traffic load. The number of BBUs calculated using our DRAC approach can achieve up to $80 \%$ and $87.5 \%$ savings of BBUs compared to traditional RAN scenarios. Table III presents the average number of BBUs and on RRHs as well as the minimum and maximum average number of handled RRHs per BBU. Clearly, our DRAC approach achieves more BBUs savings to handle the same volume of traffic load with reduced number of RRHs. This not only improves the network capacity, since many cells will be managed by the same BBU, but also creates less overhead in fronthaul links of the network.

5) Number of changed states: Finally, Fig. 5 investigates the stability of our approach by computing the impact of users' arrival rate on the number of changed states in the network. Here, "state" refers to the user allocation vectors (i.e., PRB and power allocation) after solving the optimization problem in (20) at each new user arrival. As shown in this figure, we can see that the system encounters minimal state changes - up to $9 \%$ and $10.1 \%$ for low and high SINR threshold cases, respectively, which highlights the stability of our proposed DRAC approach.

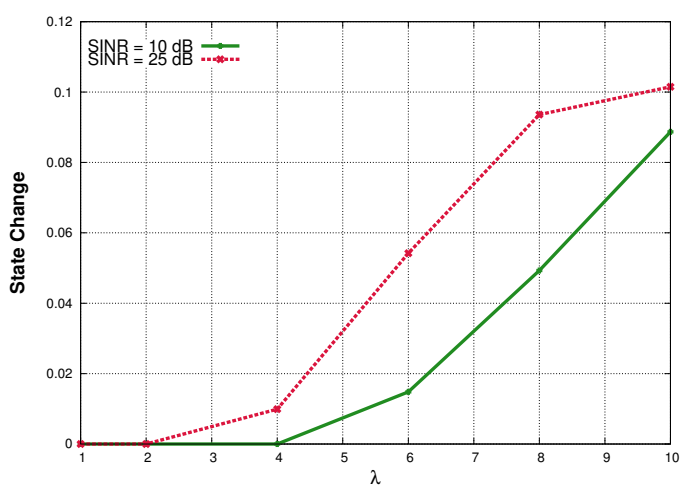

Fig. 5: Number of changed states at each resolution

\section{CONCLUSION}

In this paper, we have proposed DRAC, a novel two-stage design for Cloud-Radio Access Networks to address, firstly, the resource allocation and power minimization problems subject to power and SINR constraints, as well as the BBURRH assignment problem. Our framework can determine at each UE's arrival the optimal number of RRHs to be turned on and the number of needed BBUs to handle the whole traffic load. Through simulations, we demonstrated that our proposal achieves a good tradeoff between transmit power minimization and high throughput satisfaction rate for mobile users compared to state-of-the-art schemes. It has also been shown that our method result in significant BBU savings with maximum number of handled RRHs per BBU. This result will consequently lead in less overhead in fronthaul links as well as less interference issues between cells.

\section{ACKNOWLEDGMENT}

This work was partially supported by the ANR ABCD project and the FUI ELASTIC Network project.

\section{REFERENCES}

[1] China Mobile Research Institute, White Paper, Version 3.0. C-RAN, The Road Towards Green RAN, December 2013.

[2] B. Haberland, F. Derakhshan, H. Grob-Lipski, R. Klotsche, W. Rehm, P. Schefczik, and M. Soellner. Radio Base Stations in the Cloud. Bell Labs Technical Journal 18(1), 129152 (2013) 2013 Alcatel-Lucent., 2013.

[3] 3GPP TS 36.211. Physical channels and modulation, 3GPP. www.3gpp.org, Sept. 2008.

[4] A. Checko, H. Christiansen, Y. Yan, L. Scolari, G. Kardaras, Michael S. Berger, and L. Dittmann. Cloud RAN for Mobile Networks - a Technology Overview. IEEE COMMUNICATIONS SURVEYS AND TUTORIALS, 2013.

[5] IBM ILOG CPLEX Optimizer Version 1 v12.6. available: http://www01.ibm.com/software/commerce/optimization/cplex-optimizer/.

[6] A. Hatoum, R. Langar, N. Aitsaadi, R. Boutaba, and G. Pujolle. Qosbased power control and resource allocation in OFDMA femtocell networks. In Global Communications Conference (GLOBECOM), 2012 IEEE, pages 5116-5122, 2012.

[7] Y. Shi, J. Zhang, and K.B. Letaief. Group sparse beamforming for green cloud-RAN. Wireless Communications, IEEE Transactions on, 2014.

[8] S. Kaneko S. Namba, T. Warabino. BBU-RRH Switching Schemes for Centralized Ran. 2012 7th International ICST Conference on Communications and Networking in China (CHINACOM), 2012.

[9] H. Holm A. Checko and H. Christiansen. Optimizing small cell deployment by the use of C-RANs. European Wireless 2014, VDE VERLAG GMBH, Berlin, Offenbach, Germany, 2014.

[10] S. Namba, T. Matsunaka, T. Warabino, S. Kaneko, and Y. Kishi. ColonyRAN Architecture for Future Cellular Network. Future Network Mobile Summit (FutureNetw), pp. 1 8, July 2012.

[11] X. Huang Y. Yu, E. Dutkiewicz and M. Mueck. Downlink Resource Allocation for Next Generation Wireless Networks with Inter-Cell Interference. IEEE TRANSACTIONS ON WIRELESS COMMUNICATIONS, VOL. 12, NO. 4, April 2013.

[12] Y. Shi, Y. T. Hou, S. Kompella, and H. D. Sherali. Maximizing capacity in multihop cognitive radio networks under the SINR model. IEEE Transactions on Mobile Computing, 10(7), July 2011.

[13] S. Leyffer. Mixed integer nonlinear programming. Berlin, Germany: Springer-Verlag, 2012.

[14] G. B. Mathews. On the partition of numbers. Proc. London Math. Soc., vol. 28, p. 486-490, 1897.

[15] J. E. Mitchell. Branch-and-Cut Algorithms for Combinatorial Optimization Problems. Mathematical Sciences Rensselaer Polytechnic Institute Troy, NY, USA, 1991.

[16] J. Löfberg. YALMIP : A toolbox for modeling and optimization in MATLAB. In Proceedings of the CACSD Conference, Taipei, Taiwan, 2004. 